\title{
Bimetric Gravity Theory, Varying Speed of Light and the Dimming of Supernovae
}

\author{
J. W. Moffat \\ Department of Physics, University of Toronto, Toronto, Ontario M5S 1A\%, \\ Canada \\ and \\ Perimeter Institute for Theoretical Physics, Waterloo, Ontario N2J 2W9, \\ Canada
}

\begin{abstract}
In the bimetric scalar-tensor gravitational theory there are two frames associated with the two metrics $\hat{g}_{\mu \nu}$ and $g_{\mu \nu}$, which are linked by the gradients of a scalar field $\phi$. The choice of a comoving frame for the metric $\hat{g}_{\mu \nu}$ or $g_{\mu \nu}$ has fundamental physical consequences for local observers in either metric spacetimes, while maintaining diffeomorphism invariance. When the metric $g_{\mu \nu}$ is chosen to be associated with comoving coordinates, then the speed of light varies in the frame with the metric $\hat{g}_{\mu \nu}$. Observers in this frame see the dimming of supernovae because of the increase of the luminosity distance versus red shift, due to an increasing speed of light in the past universe. Moreover, in this frame the scalar field $\phi$ describes a dark energy component in the Friedmann equation for the cosmic scale without acceleration. If we choose $\hat{g}_{\mu \nu}$ to be associated with comoving coordinates, then an observer in the $g_{\mu \nu}$ metric frame will observe the universe to be accelerating and the supernovae will appear to be farther away. The theory predicts that the gravitational constant $G$ can vary in spacetime, while the fine-structure constant $\alpha=e^{2} / \hbar c$ does not vary. The problem of cosmological horizons as viewed in the two frames is discussed.
\end{abstract}

e-mail: john.moffat@utoronto.ca 


\section{Introduction}

Einstein's special relativity is based on the two postulates:

1. All physical laws are invariant with respect to inertial reference frames;

2. The speed of light is a universal constant with respect to all inertial reference frames.

The first postulate states that physical phenomena have the same appearance in all inertial reference frames. One obtains the same results for all measurable quantities and clocks are synchronized by means of two-way speed of light measurements. The second postulate states that there is no preferred reference frame and that globally, physical laws appear the same in all inertial frames. For a description of a consistent special relativity theory, it is sufficient to just adopt postulate (1) [1]. However, the speed of light is still a constant but this constant can vary from one reference frame to another. The spacetime coordinates are defined so that the one-way speed of light is constant.

In a Galilean spacetime the physical existence of an absolute time is postulated. The global concept of past, present and future is the same in all inertial frames. Simultaneous events in spacetime can occur in all reference frames. There exists a unique separation of past and future events. The speed of light is only constant in the Newtonian rest frame, which is a preferred frame of reference. Electromagnetic waves are limited not to exceed the speed of light.

The first postulate of Einstein's special relativity is well established by local experiments, whereas the second one is not. In contrast to Galilean relativity with its absolute time, simultaneity is not an absolute concept, but a relative one, depending on the motion of the observer. However, the first postulate of Einstein's special relativity may not be so well established at the scale of cosmology or at short distances.

Although the local Lorentz invariance of the laws of physics is a mathematical and physically attractive idea, there have been several reasons to be concerned about its generality at a fundamental level. It should be emphasized that there is no experiment that determines the one-way speed of light, for this would suppose that we can synchronize physical clocks by some way other than by finite speed of light signals. The main reason to be concerned with the generality of Einstein's special relativity is the conflict between the 
two cornerstones of modern physics, General relativity (GR) and quantum mechanics.

In GR the concept of time disappears as a physically important quantity, because of the reparameterization invariance of the theory. This has led to considerable research in attempts to understand how to construct a consistent theory of quantum gravity [2]. When we attempt to "quantize" spacetime, we find it difficult to maintain our classical ideas about local causality and special relativity associated with the Minkowski lightcone. Moreover, in quantum mechanics time is an external parameter, whereas in special relativity space and time are on an equal footing.

In cosmology, a comoving coordinate time does appear in the framework of a Friedmann, Robertson and Walker (FRW) spacetime, in which the time $t$ appears as a universal time which measures the lifetime of the universe. However, the FRW metric is subject to a diffeomorphism reparameterization transformation to non-comoving coordinates and the notion of a "universal" absolute time disappears.

The idea that a variable speed of light can solve the initial value problems of standard big-bang cosmology has received considerable attention [3, 4, 5]. From our considerations of relativity theory, we learn that it is not possible to have a varying speed of light, without modifying Einstein's formulation of special relativity and GR.

In our first treatment of a varying speed of light theory [3, 4], we introduced a formalism for spontaneously breaking the local Lorentz invariance of the vacuum within GR. The symmetry of the homogeneous Lorentz group $S O(3,1)$ was spontaneously broken to the Galilean group $O(3)$ in the early universe. This introduced the concept of an "absolute" time and a formulation of quantum gravity that did not find itself in conflict with the concept of time in quantum mechanics. As the universe expanded, a phase transition occurred that reinstated the four-dimensional symmetry of the homogenous Lorentz group. However, the speed of light was only allowed to change discontinuously from one constant value near the beginning of the universe to a much smaller value corresponding to the presently measured value of the speed of light $c_{0}=299792458 \mathrm{~m} / \mathrm{s}$. A consistent treatment of a varying speed of light within a gravitational theory was lacking. In order to remedy this problem, a bimetric gravity theory (BGT) was constructed, which provided a consistent diffeomorphism invariant formalism for descibing varying speed of light phenomena [6, 7, 8, 9, 10]. 
In the following, we shall focus on the scalar-tensor bimetric theory [0], 9]. The theory can be the preliminary stage for a quantum gravity theory that does not conflict with some of the basic properties of quantum mechanics. The physical effects of having two different spacetime metrics may be expected to be observationally detectable only at cosmological scales, when dark matter and dark energy dominate the gravitational effects, or at short distances. The BGT satisfies a matter conservation law that assures that matter test particles travel along geodesics and do not violate the weak equivalence principle, which is observationally well-tested [12]. Recently, the predictions of a primordial fluctuation spectrum were obtained from the scalar-tensor bimetric gravity theory and compared to the CMB data [11].

The observations of supernovae (SNe Ia) at red shifts $0.35<z<1.75$ have shown that they appear to be farther away and therefore fainter than is to be expected from the standard decelerating model of the universe [13]. This has led to the introduction of dark energy models which generate an accelerating expansion of the universe, based on either a cosmological constant or a form of quintessence [14]. The major problem with these models is that they lead to a coincidence problem, and a fine-tuned tiny mass smaller than the Hubble parameter, $m_{Q} \sim 10^{-33} \mathrm{eV}$, and small couplings to visible matter which must satisfy fifth-force constraints [15]. Moreover, an eternally accelerating universe will produce de Sitter event horizons that cause problems for string theory and quantum gravity theories [16].

Recently, an alternative explanation of the SNe Ia observations was proposed without the acceleration caused by dark energy, based on the idea of flavor oscillations [17]. An axion and a photon mixing causes an attenuation of the photon flux from distant systems. The model postulates an axion mass $m_{A} \sim 10^{-16} \mathrm{eV}$ and a coupling with a mass scale $M_{S} \sim 10^{11} \mathrm{GeV}$. A higher-dimensional model with leakage into the higher dimension has also been proposed as an alternative to the standard cosmological constant or quintessence models [18].

In the following, we shall consider the problem of the dimming of supernovae within the BGT in the variable speed of light (VSL) frame.

The Friedmann equation obtained from the field equations of BGT in the VSL frame leads to dark matter and dark energy components which give the experimentally observed result $\Omega_{\text {Tot }} \sim 1$, without generating an accelerating expansion of the universe. The observed dimming of the SNe Ia is affected by an increase of the red shift by 10\%-15\%, due to an increase in the speed of light in the past, which increases the luminosity distance to the supernovae. 
We consider the consequences for other fundamental "constants" of nature such as Newton's constant $G$ and the fine-structure constant $\alpha=e^{2} / \hbar c$ and discuss the problem of cosmological horizons in BGT.

\section{Bimetric Scalar-Tensor Gravity}

Our scalar-tensor bimetric gravity theory (BGT) [7, 9] is described by two metrics called the "matter metric" $\hat{g}_{\mu \nu}$ and the "gravitational metric" $g_{\mu \nu}$, respectively, connected by the "biscalar" field $\phi$ through the equation

$$
\hat{g}_{\mu \nu}=g_{\mu \nu}+B \partial_{\mu} \phi \partial_{\nu} \phi,
$$

where the constant $B$ has dimensions of [length $]^{2}$ and is chosen to be positive. The inverse metrics $\hat{g}^{\mu \nu}$ and $g^{\mu \nu}$ satisfy

$$
\hat{g}^{\mu \alpha} \hat{g}_{\nu \alpha}=\delta^{\mu}{ }_{\nu}, \quad g^{\mu \alpha} g_{\nu \alpha}=\delta^{\mu}{ }_{\nu} .
$$

We shall restrict ourselves to the physical non-degenerate case: $\operatorname{Det}\left(\hat{g}_{\mu \nu}\right) \neq$ 0 . The metric $\hat{g}_{\mu \nu}$ is used to construct the matter action and can be said to be the geometry on which matter fields propagate. It is the combination of the gravitational metric and the biscalar field that we consider as being the gravitational fields of our theory. One of the satisfactory features of the theory is that the formalism is generally covariant (diffeomorphism invariant), which guarantees that basic properties of the theoretical structure are consistent.

The model that we introduced in [7, 9] consisted in a self-gravitating

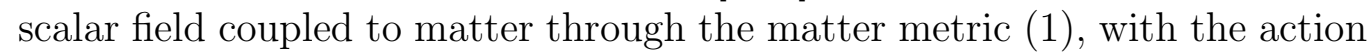

$$
S=S_{\text {grav }}+S_{\phi}+\hat{S}_{\mathrm{M}},
$$

where

$$
S_{\text {grav }}=-\frac{1}{\kappa} \int d \mu(R[g]+2 \Lambda),
$$

$\kappa=16 \pi G / c_{0}^{4}, \Lambda$ is the cosmological constant, and we employ a metric with signature $(+,-,-,-)$. We will write, for example, $d \mu=d^{4} x \sqrt{-g}$ and $\mu=$ $\sqrt{-g}$ for the metric density related to the gravitational metric $g_{\mu \nu}$, and similar definitions of $d \hat{\mu}$ and $\hat{\mu}$ in terms of the matter metric $\hat{g}_{\mu \nu}$. The minimallycoupled scalar field action is given by

$$
S_{\phi}=\frac{1}{\kappa} \int d \mu\left[\frac{1}{2} g^{\mu \nu} \partial_{\mu} \phi \partial_{\nu} \phi-V(\phi)\right],
$$


where the scalar field $\phi$ has been chosen to be dimensionless. The energymomentum tensor for the scalar field that we will use is given by

$$
T_{\phi}^{\mu \nu}=\frac{1}{\kappa}\left[g^{\mu \alpha} g^{\nu \beta} \partial_{\alpha} \phi \partial_{\beta} \phi-\frac{1}{2} g^{\mu \nu} g^{\alpha \beta} \partial_{\alpha} \phi \partial_{\beta} \phi+g^{\mu \nu} V(\phi)\right],
$$

and is the variation of the scalar field action with respect to the gravitational metric:

$$
\frac{\delta S_{\phi}}{\delta g_{\mu \nu}}=-\frac{1}{2} \mu T_{\phi}^{\mu \nu}
$$

We shall use the metric (囵) to construct the matter action $\hat{S}_{\mathrm{M}}$, resulting in the identification of $\hat{g}_{\mu \nu}$ as the metric that provides the arena on which matter fields interact. The matter action $\hat{S}_{\mathrm{M}}\left[\psi^{I}\right]=\hat{S}_{\mathrm{M}}\left[\hat{g}, \psi^{I}\right]$, where $\psi^{I}$ represents all the matter fields in spacetime. The energy-momentum tensor

$$
\frac{\delta S_{\mathrm{M}}}{\delta \hat{g}_{\mu \nu}}=-\frac{1}{2} \hat{\mu} \hat{T}^{\mu \nu}
$$

satisfies the conservation laws

$$
\hat{\nabla}_{\nu}\left[\hat{\mu} \hat{T}^{\mu \nu}\right]=0
$$

This follows as a consequence of the matter field equations only [7, 9]. Here, $\hat{\nabla}_{\mu}$ is the metric compatible covariant derivative determined by the matter metric: $\hat{\nabla}_{\alpha} \hat{g}_{\mu \nu}=0$.

The gravitational field equations are given by [7, 9]:

$$
G^{\mu \nu}=\Lambda g^{\mu \nu}+\frac{\kappa}{2} T_{\phi}^{\mu \nu}+\frac{\kappa}{2} \frac{\hat{\mu}}{\mu} \hat{T}^{\mu \nu}
$$

The scalar field wave equation is

$$
\nabla_{\mu} \nabla^{\mu} \phi+V^{\prime}(\phi)-\kappa \frac{\hat{\mu}}{\mu} \hat{T}^{\mu \nu} \hat{\nabla}_{\mu} \hat{\nabla}_{\nu} \phi=0
$$

where $\nabla_{\mu}$ is the covariant derivative with respect to the metric $g_{\mu \nu}$.

From the definition (1), we obtain the inverses

$$
\hat{g}^{\mu \nu}=g^{\mu \nu}-\frac{B}{I} \nabla^{\mu} \phi \nabla^{\nu} \phi,
$$


and

$$
g^{\mu \nu}=\hat{g}^{\mu \nu}+\frac{B}{K} \hat{\nabla}^{\mu} \phi \hat{\nabla}^{\nu} \phi
$$

where

$$
I=1+B g^{\mu \nu} \partial_{\mu} \phi \partial_{\nu} \phi, \quad K=1-B \hat{g}^{\mu \nu} \partial_{\mu} \phi \partial_{\nu} \phi .
$$

It follows that $I K=1$ and we have defined $\nabla^{\mu} \phi=g^{\mu \nu} \partial_{\nu} \phi$ and

$$
\hat{\nabla}^{\mu} \phi=\hat{g}^{\mu \nu} \partial_{\nu} \phi=K \nabla^{\mu} \phi .
$$

We will assume a perfect fluid form for the matter fields

$$
\hat{T}^{\mu \nu}=\left(\rho+\frac{p}{c_{0}^{2}}\right) \hat{u}^{\mu} \hat{u}^{\nu}-p \hat{g}^{\mu \nu}
$$

where $\hat{g}_{\mu \nu} \hat{u}^{\mu} \hat{u}^{\nu}=1$ and $\hat{u}^{\mu}=d x^{\mu} / d \hat{s}$.

A fundamental feature of the BGT is that there is a separate frame associated with each metric, and each frame or metric has its own light cone. Because of the biscalar field linkage between the two frames, the two light cones cannot be physically identical. If we choose a small patch of spacetime in the "gravitational" frame with the metric $g_{\mu \nu}$, then we can locally make the $g_{\mu \nu}$ metric equal the flat, Minkowski metric, i.e. $g_{\mu \nu}=\eta_{\mu \nu}$ where $\eta_{\mu \nu}=\operatorname{diag}(1,-1,-1,-1)$. This yields

$$
d s^{2}=\eta_{\mu \nu} d x^{\mu} d x^{\nu}
$$

However, from (11) we obtain

$$
d \hat{s}^{2}=\eta_{\mu \nu} d x^{\mu} d x^{\nu}+B \partial_{\mu} \phi \partial_{\nu} \phi d x^{\mu} d x^{\nu}
$$

If we perform a local Lorentz transformation

$$
x^{\prime \mu}=\Lambda_{\nu}^{\alpha} x^{\nu},
$$

where $\Lambda^{\alpha}{ }_{\nu}$ are constant tensor coefficients that satisfy

$$
\Lambda_{\mu}^{\alpha} \Lambda_{\alpha \nu}=\eta_{\mu \nu}
$$

then (17) and (18) both remain form invariant. This follows if we have $V_{\mu}=\partial_{\mu} \phi$, where $V_{\mu}^{\prime}=\Lambda_{\mu}{ }^{\alpha} V_{\alpha}$ under a Lorentz transformation. If the light cone equation $d s^{2}=0$ is satisfied, then $d \hat{s}^{2} \neq 0$ along the same light cone unless $\phi=0$. However, $d \hat{s}^{2}=0$ along an expanding light cone that encloses 
the light cone $d s^{2}=0$. We observe that whereas we can transform away the Christoffel symbols $\Gamma_{\mu \nu}^{\lambda}$ at a spacetime point, we cannot transform away the scalar field $\phi$ at this point, nor the Christoffel symbol $\hat{\Gamma}_{\mu \nu}^{\lambda}$.

If we choose the vacuum expectation value $\mathcal{V}_{\mu}=<V_{\mu}>_{0} \neq 0$, then the gradient of the scalar field $\phi$ acts as a spontaneous symmetry breaking field, so that it is not possible to maintain the local Lorentz invariance of the vacuum states of both $\hat{g}_{\mu \nu}$ and $g_{\mu \nu}$ metrics. Thus, a preferred frame is chosen under the spontaneous symmetry breaking of the vacuum, since $\mathcal{V}_{\mu}$ picks out a preferred direction in spacetime. This frame can correspond to a timelike vector $\mathcal{V}_{\mu}=(\mathcal{V}, 0,0,0)$, leading to a spontaneous breaking of the homogeneous Lorentz group $S O(3,1) \rightarrow O(3)$ i.e. only the symmetry group of rotations would be preserved. In GR there is only one rigid light cone and one metric and local Lorentz invariance is strictly maintained.

In the BGT developed in earlier papers, we assumed that in the VSL frame all particles move with the the speed of light $c(t)$. We can generalize this theory, so that each particle has its own light cone by adopting the metric definition:

$$
\hat{g}_{I \mu \nu}=g_{\mu \nu}+B_{I} \partial_{\mu} \phi_{I} \partial_{\nu} \phi_{I}
$$

where the label $I$ denotes the specific particle with its associated speed $c_{I}(t)$.

\section{Bimetric Gravity Cosmology}

Let us now consider a cosmological scenario, imposing homogeneity and isotropy on spacetime and writing the "gravitational frame" metric $g_{\mu \nu}$ in comoving form

$$
d s^{2}=c_{0}^{2} d t^{2}-R^{2}(t)\left[\frac{d r^{2}}{1-k r^{2}}+r^{2} d \theta^{2}+r^{2} \sin ^{2} \theta d \phi^{2}\right]
$$

where we employ a dimensionless radial variable $r$ and $k=0, \pm 1$ for flat, closed and open hyperbolic spatial topologies, respectively. Then, the metric in what we call the VSL frame takes the form

$$
d \hat{s}^{2}=c^{2}(t) d t^{2}-R^{2}(t)\left[\frac{d r^{2}}{1-k r^{2}}+r^{2} d \theta^{2}+r^{2} \sin ^{2} \theta d \phi^{2}\right]
$$

where

$$
c(t)=c_{0} I^{1 / 2}
$$


and

$$
I=1+\frac{B}{c_{0}^{2}} \dot{\phi}^{2}
$$

An overdot indicates a derivative with respect to the time variable $t$.

The matter stress-energy tensor is

$$
\hat{T}^{00}=\frac{\rho}{I}, \quad \hat{T}^{0 i}=0, \quad \hat{T}^{i j}=\frac{p}{R^{2}} \gamma^{i j},
$$

where $\gamma^{i j}(i, j=1,2,3)$ denotes the spatial metric. The conservation laws become

$$
\dot{\rho}+3 H\left(\rho+\frac{p}{c_{0}^{2}}\right)=0
$$

where $H=\dot{R} / R$ is the Hubble function.

We see that in the VSL frame the speed of light depends on time, whereas in the variable speed of gravitational waves (VSGW) frame, the speed of light is constant and the speed of gravitational waves depends on time. The latter frame is arrived at by writing the matter metric $\hat{g}_{\mu \nu}$ in comoving form with constant $c_{0}$ :

$$
d \hat{s}^{2}=c_{0}^{2} d t^{2}-R^{2}(t)\left[\frac{d r^{2}}{1-k r^{2}}+r^{2} d \theta^{2}+r^{2} \sin ^{2} \theta d \phi^{2}\right] .
$$

We now obtain

$$
d s^{2}=v_{g}^{2} d t^{2}-R^{2}(t)\left[\frac{d r^{2}}{1-k r^{2}}+r^{2} d \theta^{2}+r^{2} \sin ^{2} \theta d \phi^{2}\right]
$$

where $v_{g}$ is the speed of gravitational waves

$$
v_{g}(t)=c_{0} K^{1 / 2}
$$

and

$$
K=1-\frac{B}{c_{0}^{2}} \dot{\phi}^{2}
$$

We see that the speed of gravitational waves $v_{g}$ in the VSGW frame is predicted for $B>0$ to be smaller than the presently measured speed of light $c_{0}$.

The laws of physics will be interpreted differently by observers, depending upon whether they perform experiments in the VSL frame or the VSGW frame. 
The Friedmann equation in the VSL frame is given by

$$
H^{2}+\frac{k c_{0}^{2}}{R^{2}}=\frac{8 \pi G}{3 I^{1 / 2}} \rho+\frac{1}{3} c_{0}^{2} \Lambda+\frac{1}{6} \rho_{\phi},
$$

where

$$
\rho_{\phi}=\frac{1}{2} \dot{\phi}^{2}+c_{0}^{2} V(\phi)
$$

The remaining equation is

$$
\frac{\ddot{R}}{R}=-\frac{4 \pi G}{3 I^{1 / 2}}\left(\rho+3 I \frac{p}{c_{0}^{2}}\right)+\frac{1}{3} c_{0}^{2} \Lambda-\frac{1}{12}\left(\rho_{\phi}+3 p_{\phi}\right),
$$

where

$$
p_{\phi}=\frac{1}{2} \dot{\phi}^{2}-c_{0}^{2} V(\phi) .
$$

The scalar field equation (11) in the VSL frame is

$$
\frac{1}{c_{0}^{2}}\left(1-\frac{16 \pi G B}{c_{0}^{2} I^{3 / 2}} \rho\right) \ddot{\phi}+\frac{3}{c_{0}^{2}} H \dot{\phi}\left(1+\frac{16 \pi G B}{c_{0}^{4} I^{1 / 2}} p\right)+V^{\prime}(\phi)=0 .
$$

The Friedmann equation in the VSGW frame is given by

$$
H^{2}+\frac{c_{0}^{2} k K}{R^{2}}=\frac{8 \pi G}{3} K^{3 / 2} \rho+\frac{1}{3} c_{0}^{2} \Lambda K+\frac{1}{6} \tilde{\rho}_{\phi} .
$$

where we have defined

$$
\tilde{\rho}_{\phi}=\frac{1}{2} \dot{\phi}^{2}+c_{0}^{2} K V(\phi), \quad \tilde{p}_{\phi}=\frac{1}{2} \dot{\phi}^{2}-c_{0}^{2} K V(\phi) .
$$

The remaining equation is

$$
\frac{\ddot{R}}{R}=-\frac{4 \pi G}{3}\left(K^{3 / 2} \rho+\frac{3}{c_{0}^{2}} K^{1 / 2} p\right)+\frac{1}{3} c_{0}^{2} \Lambda K-\frac{1}{12}\left(\tilde{\rho}_{\phi}+3 \tilde{p}_{\phi}\right)+\frac{1}{2} \frac{\dot{K}}{K} H .
$$

The scalar wave equation in this frame is given by

$$
\frac{1}{c_{0}^{2}}\left(1-\frac{16 \pi G B}{c_{0}^{2}} K^{3 / 2} \rho\right) \ddot{\phi}+\frac{3 K}{c_{0}^{2}} H \dot{\phi}\left(1+\frac{16 \pi G B}{c_{0}^{4}} K^{1 / 2} p\right)+K^{2} V^{\prime}(\phi)=0 .
$$




\section{Calculation of the Red Shift}

By adopting the VSL frame and setting $d \hat{s}^{2}=0$ in (23), we obtain for fixed $\theta$ and $\phi$ :

$$
\int_{t_{1}}^{t_{0}} \frac{d t c(t)}{R(t)}=\int_{0}^{r_{1}} \frac{d r}{\sqrt{1-k r^{2}}}=f\left(r_{1}\right)=\text { const. }
$$

where $t_{1}$ denotes the time a light wave leaves a supernova or galaxy and $t_{0}$ denotes the time when it reaches us on earth. If the next wave crest leaves $r_{1}$ at time $t_{1}+\delta t_{1}$, it will arrive on earth at time $t_{0}+\delta t_{0}$ giving

$$
\int_{t_{1}+\delta t_{1}}^{t_{0}+\delta t_{0}} \frac{d t c(t)}{R(t)}=f\left(r_{1}\right) .
$$

Subtracting (41) from (42) and considering that $R$ and $c$ vary little during the period of a light signal, we obtain

$$
\frac{c\left(t_{0}\right) \delta t_{0}}{R\left(t_{0}\right)}=\frac{c\left(t_{1}\right) \delta t_{1}}{R\left(t_{1}\right)}
$$

The frequency at emission $\nu_{1}$ is then related to the observed frequency $\nu_{0}$ by

$$
\frac{\nu_{0}}{\nu_{1}}=\frac{\delta t_{1}}{\delta t_{0}}=\frac{c\left(t_{0}\right)}{c\left(t_{1}\right)} \frac{R\left(t_{1}\right)}{R\left(t_{0}\right)} .
$$

The red shift

$$
z=\frac{\lambda_{0}-\lambda_{1}}{\lambda_{1}}
$$

is then given by

$$
z=\frac{c\left(t_{1}\right)}{c\left(t_{0}\right)} \frac{R\left(t_{0}\right)}{R\left(t_{1}\right)}-1
$$

where we have

$$
\lambda(t) \nu(t)=c_{0},
$$

so that $\lambda_{0} / \lambda_{1}=\nu_{1} / \nu_{0}$. This follows from the relation

$$
\hat{k}^{\mu} \hat{k}_{\mu}=\hat{g}^{\mu \alpha} k_{\alpha} \hat{g}_{\mu \beta} k^{\beta}=k_{\beta} k^{\beta}=0,
$$

where we have used (2) and $k^{\mu}=\left(\nu / c_{0}, 1 / \vec{\lambda}\right)$ and $\lambda=|\vec{\lambda}|$.

We see that if $c\left(t_{1}\right)>c\left(t_{0}\right)$, then the observed red shift will appear to be larger due to the speed of light in the past being bigger than the presently observed speed $c\left(t_{0}\right)=c_{0}$ in an expanding universe with $R\left(t_{0}\right)>R\left(t_{1}\right)$. 


\section{Apparent Luminosity and Luminosity Dis- tance}

The apparent luminosity $l$ is the power per unit mirror area in our VSL frame 19:

$$
l \equiv \frac{P}{A}=L\left(\frac{c^{2}\left(t_{0}\right)}{c^{2}\left(t_{1}\right)}\right)\left(\frac{R^{2}\left(t_{1}\right)}{R^{2}\left(t_{0}\right)}\right)\left(\frac{1}{4 \pi R^{2}\left(t_{0}\right) r_{1}^{2}}\right),
$$

where $L$ is the absolute luminosity, $A$ is the proper area of the mirror and $P$ is the total power emitted at the source. The luminosity distance of a light source in Euclidean space is

$$
d_{L}=\left(\frac{L}{4 \pi l}\right)^{1 / 2}
$$

which leads to the expression

$$
d_{L}=\left(\frac{c\left(t_{1}\right)}{c\left(t_{0}\right)}\right)\left(\frac{R^{2}\left(t_{0}\right) r_{1}}{R\left(t_{1}\right)}\right) .
$$

Let us expand $R(t)$ as

$$
R(t)=R\left(t_{0}\right)\left[1+H_{0}\left(t-t_{0}\right)-\frac{1}{2} q_{0} H_{0}^{2}\left(t-t_{0}\right)^{2}+\ldots\right]
$$

where

$$
H_{0}=\frac{\dot{R}\left(t_{0}\right)}{R\left(t_{0}\right)}, \quad q_{0}=-\frac{\ddot{R}\left(t_{0}\right) R\left(t_{0}\right)}{\dot{R}^{2}\left(t_{0}\right)} .
$$

We also expand $c(t)$ in the power series

$$
c(t)=c\left(t_{0}\right)\left[1+D_{0}\left(t-t_{0}\right)+\frac{1}{2} Q_{0} D_{0}^{2}\left(t-t_{0}\right)^{2}+\ldots\right]
$$

where

$$
D_{0}=-\frac{\dot{c}\left(t_{0}\right)}{c\left(t_{0}\right)}, \quad Q_{0}=-\frac{\ddot{c}\left(t_{0}\right) c\left(t_{0}\right)}{\dot{c}^{2}\left(t_{0}\right)} .
$$

The negative signs of the coefficients $D_{0}$ and $Q_{0}$ are chosen to yield a decreasing speed of light as the universe expands. From (46), we get using (52) and (54) the red shift

$$
z=\left(H_{0}-D_{0}\right)\left(t_{0}-t_{1}\right)+\left[\left(1+\frac{1}{2} q_{0}\right) H_{0}^{2}+\frac{1}{2} Q_{0} D_{0}^{2}-D_{0} H_{0}\right]\left(t_{0}-t_{1}\right)^{2}+\ldots
$$


Inverting this equation we get

$$
t_{0}-t_{1}=\left(\frac{1}{H_{0}-D_{0}}\right) z-\left(\frac{1}{H_{0}-D_{0}}\right)^{3}\left[\left(1+\frac{1}{2} q_{0}\right) H_{0}^{2}+\frac{1}{2} Q_{0} D_{0}^{2}-D_{0} H_{0}\right] z^{2}+\ldots
$$

From (41), we obtain

$$
\left(\frac{c\left(t_{0}\right)}{R\left(t_{0}\right)}\right)\left[t_{0}-t_{1}+\frac{1}{2}\left(H_{0}-D_{0}\right)\left(t_{0}-t_{1}\right)^{2}+. .\right]=r_{1}+O\left(r_{1}^{3}\right) .
$$

This gives the result for $r_{1}$ :

$$
\begin{gathered}
r_{1}=\left(\frac{c\left(t_{0}\right)}{R\left(t_{0}\right)}\right)\left\{\left(\frac{1}{H_{0}-D_{0}}\right) z-\left(\frac{1}{H_{0}-D_{0}}\right)^{3}\left[\left(1+\frac{1}{2} q_{0}\right) H_{0}^{2}+\frac{1}{2} Q_{0} D_{0}^{2}-D_{0} H_{0}\right] z^{2}\right. \\
\left.+\frac{1}{2}\left(\frac{1}{H_{0}-D_{0}}\right) z^{2}+\ldots\right\}
\end{gathered}
$$

From (46) and (51), we find

$$
\begin{gathered}
d_{L}=c\left(t_{0}\right) r_{1}(1+z) \\
=\left(\frac{c\left(t_{0}\right)}{H_{0}-D_{0}}\right)\left\{z-\left(\frac{1}{H_{0}-D_{0}}\right)^{2}\left[\left(1+\frac{1}{2} q_{0}\right) H_{0}^{2}+\frac{1}{2} Q_{0} D_{0}^{2}-D_{0} H_{0}\right.\right. \\
\left.\left.-\frac{3}{2}\left(H_{0}-D_{0}\right)^{2}\right] z^{2}+\ldots\right\} .
\end{gathered}
$$

In the limit that $D_{0}=Q_{0}=0$, we obtain the correct GR limit

$$
d_{L}=\left(\frac{c\left(t_{0}\right)}{H_{0}}\right)\left[z+\frac{1}{2}\left(1-q_{0}\right) z^{2}+\ldots\right] .
$$

We now obtain the formula for the apparent luminosity

$$
\begin{aligned}
l \equiv \frac{L}{4 \pi d_{L}^{2}}= & \left(\frac{L}{4 \pi z^{2} c^{2}\left(t_{0}\right)}\right)\left(H_{0}-D_{0}\right)^{2}\left\{1+\left(\frac{1}{H_{0}-D_{0}}\right)^{2}\left[\left(1+\frac{1}{2} q_{0}\right) H_{0}^{2}\right.\right. \\
& \left.\left.+\frac{1}{2} Q_{0} D_{0}^{2}-D_{0} H_{0}-\frac{3}{2}\left(H_{0}-D_{0}\right)^{2}\right] z^{2}+\ldots\right\} .
\end{aligned}
$$




\section{Dimming of Supernovae}

Let us write the Friedmann equation (32) as

$$
\Omega_{0 m}+\Omega_{0 k}+\Omega_{0 \Lambda}+\Omega_{0 \phi}=1 \text {, }
$$

where

$$
\begin{gathered}
\Omega_{0 m}=\frac{8 \pi G \rho_{0 m}}{3 H_{0}^{2} I_{0}^{1 / 2}}, \quad \Omega_{0 k}=-\frac{c_{0}^{2} k}{R_{0}^{2} H_{0}^{2}}, \quad \Omega_{0 \Lambda}=\frac{c_{0}^{2} \Lambda}{3 H_{0}^{2}}, \\
\Omega_{0 \phi}=\frac{\rho_{0 \phi}}{6 H_{0}^{2}},
\end{gathered}
$$

where $\Omega_{0}, \rho_{0}, \phi_{0}$ and $H_{0}$ denote the present values of the corresponding quantities.

Choosing the values

$$
\Omega_{0 m}=0.28, \quad \Omega_{0 k}=0, \quad \Omega_{0 \Lambda}=0, \quad \Omega_{0 \phi}=0.72,
$$

we obtain a fit to the supernovae data with $q_{0}=\frac{1}{2}$ and $D_{0}$ and $Q_{0}$ chosen for a given red shift $z$ to give a $10-15 \%$ increase in the luminosity distance (60), corresponding to a 20-30\% decrease in the apparent luminosity observed in the supernovae data [13]. For a negligible value of the pressure $p_{m}$, we get from (34) with $\Lambda=0$ :

$$
\frac{\ddot{R}}{R}=-\left[\left(\frac{4 \pi G}{3 I_{0}^{1 / 2}}\right) \rho_{0 m}+\frac{1}{12}\left(\rho_{0 \phi}+3 p_{0 \phi}\right)\right] .
$$

We see that the scalar field $\phi$ does not produce an acceleration of the universe, when $\rho_{0 \phi}>0$ and $p_{0 \phi}>0$. The increased speed of light at the observed SNe Ia red shifts $z$ has dimmed the supernovae in accordance with the SNe Ia observations. We do not attempt here to explain why $\Lambda=0$.

The Friedmann equation (32) can be rewritten in terms of an effective gravitational constant $G_{\text {eff }}=G / \sqrt{I(t)}$, corresponding to a time-varying gravitational constant.

We see that the time variation of $G$ depends on the time derivative of the scalar field $\phi$. The cosmic time evolution of $G$ should be of the order of the expansion rate of the universe, i.e. $\dot{G} / G \sim H_{0}$, where $H_{0}=$ $100 h \mathrm{~km} \mathrm{~s}^{-1} \mathrm{Mpc}^{-1}=h \times 10^{-10} \mathrm{yr}^{-1}$ [12]. Current observations of the expansion of the universe yield $h \sim 0.7$. For $z \sim 1$ an estimate gives $\Delta t \sim$ $4.9 \times 10^{9} \mathrm{yr}$, so that we obtain $\Delta G / G \sim 0.3$. Thus, in the red shift range 
$z \sim 0.1-3$, a $10-15 \%$ change in $G$ can be accomodated and be consistent with a $10-15 \%$ change in $c(t)=c_{0}\left(1+\frac{B}{c_{0}^{2}} \dot{\phi}^{2}\right)^{1 / 2}$. However, as we approach the time of big-bang nucleosynthesis (BBN) at a red shift $z \sim 10^{9}-10^{10}$, we have $\Delta G / G \sim 10^{-9}-10^{-10}$ and the variation of $G$ with time becomes much more restrictive. This requires that for red shifts larger than $z \sim 3$, the scalar field $\phi$ should tend to a constant and $I(t) \rightarrow 1$ in order not to violate the good agreement of BBN calculations. A more detailed numerical analysis of solutions of the BGT for $z \sim 10^{9}$ is needed to determine the behavior of $I(t)$. Such an analysis will be considered in a future publication.

If we now view the expansion of the universe from within the gravitational metric or VSGW frame, then the speed of light is constant but gravitational waves will move with a speed different from $c_{0}$. From (37), we obtain the contributions to (63):

$$
\begin{gathered}
\Omega_{0 m}=\frac{8 \pi G K_{0}^{3 / 2} \rho_{0 m}}{3 H_{0}^{2}}, \quad \Omega_{0 k}=-\frac{c_{0}^{2} k K_{0}}{R_{0}^{2} H_{0}^{2}}, \quad \Omega_{0 \Lambda}=\frac{c_{0}^{2} K_{0} \Lambda}{3 H_{0}^{2}}, \\
\Omega_{0 \phi}=\frac{\tilde{\rho}_{0 \phi}}{6 H_{0}^{2}} .
\end{gathered}
$$

We obtain from (37) the deceleration parameter

$$
q=\frac{d H^{-1}}{d t}-1=-\frac{\dot{K}}{2 H K}+\frac{1}{2}\left(1+\Omega_{k}\right)+\frac{\tilde{p}_{\phi}}{4 H^{2}}+\frac{4 \pi G}{c_{0}^{2} H^{2}} K^{1 / 2} p_{m}-\frac{c_{0}^{2} K \Lambda}{2 H^{2}} .
$$

By using the scalar wave equation (40), we find that [7, 9]:

$$
\frac{\dot{K}}{2 H K}=\frac{3(1-K)\left(1+16 \pi G B K^{1 / 2} p_{m}\right)+H^{-1} B \dot{\phi} K V^{\prime}(\phi)}{1-16 \pi G B K^{3 / 2} \rho_{m} / c_{0}^{2}} .
$$

The first term in the numerator and the denominator are positive-definite whenever the metrics have the correct signature, and the second term in the numerator is positive provided that $H>0$ and $\dot{\phi} K V^{\prime}(\phi)>0$.

If we choose $p_{m} \sim 0$ and $k=\Lambda=0$, we get a fit to the current data in the VSGW frame with

$$
\Omega_{0 m}=0.28, \quad \Omega_{0 \phi}=0.72, \quad q_{0}=-0.63,
$$

where

$$
q_{0}=-\frac{\dot{K}_{0}}{2 H_{0} K_{0}}+\frac{1}{2}+\frac{\tilde{p}_{0 \phi}}{4 H_{0}^{2}}
$$


We can achieve an acceleration of the universe, if the first term on the righthand side of (71) dominates, which means that the cosmic acceleration with $\ddot{R}>0$ is caused by the dynamics of the scalar field $\phi$ with $\Lambda=0$ in the VSGW frame. Provided that $q$ turns small and positive at an earlier time in the universe's expansion, then galaxy formation can be achieved.

Thus, in the VSL frame the universe appears to be decelerating and the supernovae are dimmed because of the increase of the speed of light with increasing red shift $z$ at least up to $z \sim 3-4$, while in the VSGW frame the universe appears to be accelerating and the supernovae appear to be farther away.

We have demonstrated elsewhere, that the early universe standard horizon and flatness problems can be resolved either in the VSL frame with a varying speed of light, or within the VSGW frame with constant speed of light, but with a varying speed of gravitational waves [3, 4, 6, 7, 8, 9].

\section{Fine-Structure Constant and Energy Con- servation}

Let us consider the behavior of the fine-structure constant $\alpha=e^{2} / \hbar c$ and the energy of a system of particles in our BGT. We shall adopt the action for a charged particle moving in an electromagnetic field

$$
S_{E M}=-\int d \hat{s}\left[m c_{0}\left(\hat{u}^{\mu} \hat{u}^{\nu} \hat{g}_{\mu \nu}\right)^{1 / 2}+\frac{e_{0}}{c_{0}} \hat{u}^{\mu} \hat{A}_{\mu}\right]
$$

where

$$
F_{\mu \nu}=\partial_{\mu} A_{\nu}-\partial_{\nu} A_{\mu},
$$

is the electromagnetic field. Moreover, $\hat{F}^{\mu \nu}=\hat{g}^{\mu \alpha} \hat{g}^{\nu \beta} F_{\alpha \beta}$ is the electromagnetic field observed in the matter VSL frame, $\hat{u}^{\mu}=\hat{g}^{\mu \alpha} u_{\alpha}, \hat{A}_{\mu}=\hat{g}_{\mu \nu} A^{\nu}, m$ is the particle mass and $e_{0}$ is the constant electron charge. We have in our isotropic and homogeneous cosmology $A^{\mu}=\left(e_{0} / r, 0\right)$.

From (2) and (72), we obtain for the Coulomb energy

$$
V_{\text {Coul }}=-\frac{e_{0}^{2}}{c_{0} r} \frac{d x_{0}}{d s}
$$

where $d x_{0} / d s=\gamma c_{0}$ with $\gamma=1 /\left(1-u^{2} / c_{0}^{2}\right)$. We obtain in the nonrelativistic limit

$$
V_{\text {Coul }}=-\frac{e_{0}^{2}}{r}
$$


In particular, the fine-structure constant

$$
\alpha \equiv \frac{e^{2}}{\hbar c}=\frac{e_{0}^{2}}{\hbar c_{0}}
$$

is constant in our bimetric gravity theory. This will be true whether or not we observe $\alpha$ in the VSL frame or in the VSGW frame.

This does not conform with the observations of absorption line spectra from quasars [20], which yield

$$
\frac{\Delta \alpha}{\alpha}=-0.72 \pm 0.18 \times 10^{-5} .
$$

However, these data still require to be confirmed by an independent measurement procedure before we can rule out the constancy of $\alpha$ predicted by the BGT. If we wish to interpret the dimming of the supernovae by a $\sim 10 \%$ increase in the speed of light in the VSL frame, then we cannot simultaneously demand a similar decrease in the fine-structure constant $\alpha$, because this would strongly disagree with the Webb et al. result (77) and lead to serious violations of the weak equivalence principle experimental tests. A recent analysis of the effects of a time varying $\alpha$ in the CMB background, gives results which are consistent with no variation in $\alpha$ from the epoch of recombination to the present day, and restricts any such variation to be less than about 4\% 21]. Forthcoming MAP and Planck experiments will be able to measure variations in $\alpha$ to better than a percent accuracy.

Finally, let us consider the conservation of energy in BGT. We have from (2):

$$
\hat{p}^{\mu} \hat{p}_{\mu}=\hat{g}^{\mu \alpha} p_{\alpha} \hat{g}_{\mu \beta} p^{\beta}=p^{\mu} p_{\mu}=\left(m c_{0}\right)^{2} .
$$

From the identification $p^{\mu}=\left(E / c_{0}, \vec{p}\right)$, we obtain the standard special relativity result

$$
E=c_{0}\left[(\vec{p})^{2}+\left(m c_{0}\right)^{2}\right]^{1 / 2} .
$$

\section{Cosmological Horizons}

According to the models of quintessence [14, the dark energy of the universe is dominated by the potential $V(\phi)$ of a scalar field $\phi$, which rolls down to its minimum at $V=0$. We recall that for an equation of state $p=w \rho$ in $\mathrm{GR}$, a cosmological constant corresponds to $w=-1$, radiation domination 
to $w=\frac{1}{3}$ and matter domination to $w=0$. On the other hand, quintessence gives an equation of state with

$$
-1<w<-\frac{1}{3}
$$

while the observational evidence for a cosmological constant is given by the bound:

$$
-1<w_{\text {observed }} \leq-\frac{2}{3}
$$

In the VSL frame, the proper horizon distance is given by

$$
\delta_{H}(t)=R(t) F
$$

where

$$
F=\int_{t_{0}}^{\infty} \frac{d t^{\prime} c\left(t^{\prime}\right)}{R\left(t^{\prime}\right)} .
$$

Whenever $F$ diverges there exist no future event horizons in the spacetime geometry. On the other hand, when $F$ converges the spacetime geometry exhibits a future horizon, and events whose coordinates at time $\bar{t}$ are located beyond $\delta_{H}$ can never communicate with the observer at $r=0$.

The variation of the expansion scale factor at large $R(t)$, when the curvature becomes negligible, approaches

$$
R(t) \sim t^{2 / 3(1+w)}
$$

We now have

$$
F=c_{0} \int_{t_{0}}^{\infty} d t^{\prime} t^{\prime 2(n-1) / 3(1+w)]}
$$

where we have assumed that a solution of the BGT field equations leads to the behavior

$$
c(t) \sim c_{0} R^{n}
$$

where $n$ is some positive number. In the VSL frame with the metric $\hat{g}_{\mu \nu}$, we can choose $w>1 / 3, \Lambda=0$ and a value of $n$ such that the integral $F$ diverges and there is no future cosmological horizon. On the other hand, in the VSGW frame with the metric $g_{\mu \nu}$, the universe is accelerating with $\ddot{R}>0$, the velocity of light is constant, $c(t)=c_{0}$, and we expect that the integral $F$ will converge and allow a future cosmological horizon.

In an eternally accelerating universe, we are confronted with the difficulty of defining a consistent S-matrix description of quantum field theory and 
string/M-theories. We have shown that if we restrict ourselves to the VSL frame with a varying speed of light and a decelerating universe, then we can avoid asymptotically all future horizons associated with quintessence models and an accelerating universe. On the other hand, if we perform our experiments and theoretical calculations in the VSGW frame, then we can expect to encounter future cosmological horizons. Thus, it is advantageous for us to restrict ourselves to the VSL frame, since we can construct a viable S-matrix for quantum field theories and string/M-theories with a satisfactory asymptotic null infinity.

\section{Conclusions}

The problem of constructing a self-consistent gravity theory which is diffeomorphism invariant and allows a varying speed of light and a varying speed of gravitational waves can be solved within the BGT formalism. A proper conservation law for the matter tensor can be constructed that leads to particles moving on geodesics in the comoving $\hat{g}_{\mu \nu}$ frame. In this frame, the speed of light is constant and observers can communicate with one another by light signals. An observer in the spacetime determined by the $g_{\mu \nu}$ metric, sees the speed of gravitational waves varying with time and the universe accelerate and a future cosmological horizon is expected to exist.

On the other hand, when the metric $g_{\mu \nu}$ is chosen to be comoving, so that the speed of gravitational waves is constant, and observers will be able (in the future) to communicate with one another by means of gravitational wave signals, then an observer in the $\hat{g}_{\mu \nu}$ frame will detect a varying speed of light, a dimming of supernovae because of the increase of $c(t)$ in the past universe, and a decelerating universe without a cosmological horizon for an increasing speed of light in the future universe.

The observer in the VSL frame will be in an advantageous position when performing physical experiments, because the non-existence of a future cosmological horizon will permit the construction of a meaningful S-matrix for the scattering of particles.

An important feature of the BGT is that the dimensionless fine-structure constant $\alpha$ is constant in spacetime, so problems associated with possible violations of the weak equivalence principle tests and massive fine-tuning of vacuum quantum field theory can be avoided [22]. However, future experiments will hopefully decide whether the putative observations of a variation 
of $\alpha$ by analyses of quasar absorption line spectra are valid [20].

There are many interesting consequences of BGT that can be explored e.g. the behavior of collapsing stars and the nature of dispersion relations at high energy. It is also of interest to investigate the possible role of BGT in quantum gravity. These topics will be the subject of future invetigations.

\section{Acknowledgments}

I thank Michael Clayton for helpful and stimulating discussions. This work was supported by the Natural Sciences and Engineering Research Council of Canada.

\section{References}

[1] F. Selleri, "Theories Equivalent to Special Relativity", Frontiers of Fundamental Physics, edited by M. Barone and F. Selleri, Plenum Press, New York, 1994; J. P. Hsu and L. Hsu, Phys. Lett. A196, 1 (1994).

[2] C. J. Isham, "Prima Facie Questions in Quantum Gravity", Relativity, Classical and Quantum, edited by J. Ehlers and H. Friedrich, SpringerVerlag, Berlin (1994), gr-qc/9310031.

[3] J. W. Moffat, Int. J. Mod. Phys. D2, 351 (1993), gr-qc/9211020; J. W. Moffat, Found. of Phys. 23, 411 (1993), gr-qc/9209001.

[4] J. W. Moffat, Fluctuating Paths and Fields, Festschrift dedicated to Hagen Kleinert, edited by W. Jancke, A. Pelster, H.-J. Schmidt, and M. Bachmann, World Scientific, Singapore, p. 741, 2001, astroph/9811390.

[5] A. Albrecht and J. Magueijo, Phys. Rev. D59, 043516, (1999), astroph/9811018; J. D. Barrow, Phys. Rev. D59, 043515 (1999); J. D. Barrow and J. Magueijo, Phys. Lett. B477, 246 (1999), astro-ph/9811073 v2; R. H. Brandenberger and J. Magueijo, "Imaginative Cosmology", Invited Lectures given at the IPM School on Cosmology 1999: Large 
Scale Structure Formation, to be published by Kluwer, Dordrecht, hepph/9912247; P. P. Avelino and C. J. A. P. Martins, Phys. Lett. B459, 468 (1999), astro-ph/9906117; P. P. Avelino, C. J. A. P. Martins, and G. Roch, Phys. Lett. B483, 210 (2000), astro-ph/0001292; T. Harko and M. K. Mak, Gen. Rel. Grav. 31, 849 (1999); Class. Quant. Grav. 16, 2741 (1999); T. Harko, H. Q. Lu, K. Mak, and K. S. Cheng, Europhys. Lett. 49, 814 (2000); J. Magueijo, Phys. Rev. D62 103521 (2000), gr-qc/0007036; Phys. Rev. D63, 043502 (2001), astro-ph/0010591; J. Magueijo, H. Sandvik, and T.W. B. Kibble, hep-ph/0101155; S. H. S. Alexander and J. Magueijo, hep-th/0104093; S. Liberati, B. A. Bassett, C. Molinari-Paris, and M. Visser, Nucl. Phys. Proc. Suppl. 88, 259 (2000), astro-ph/0001481; B. A. Bassett, S. Liberati, C. Molinari-Paris, and M. Visser, Phys. Rev. D62 103518 (2000), astro-ph/0001441 v2; E. Kiritsis, JHEP, 9910:010 (1999), hep-th/9906206; S. H. S. Alexander, JHEP, 0011:017(2000), hep-th/9912037; D. Youm, Phys. Rev. D63 (2001) 125011; hep-th/0102194; Phys. Rev. D65 025008 (2002), hepth/0108073; hep-th/0108237; J. Chung, E. W. Kolb and A. Riotto, hep-ph/0008126; hep-th/0203145; C. Csaki, J. Erlich, and C. Grojean, Nucl. Phys. 604, 312 (2001), hep-th/0012143 (2000); hep-th/0102194; J. C. Niemeyer, Phys. Rev. D65, 083505 (2002), astro-phys/0111479.

[6] M. A. Clayton and J. W. Moffat, Phys. Lett. B460, 263 (1999), astroph/9812481;

[7] M. A. Clayton and J. W. Moffat, Phys. Lett. B477, 269 (2000), grqc/9910112.

[8] M. A. Clayton and J. W. Moffat, Int. J. of Mod. Phys. D11, 187 (2002), gr-qc/0003070.

[9] M. A. Clayton and J. W. Moffat, Phys. Lett. B506, 177 (2001), grqc/0101126 v2.

[10] I. T. Drummond, Phys. Rev. D63 043503 (2001), astro-ph/0008234.

[11] M. A. Clayton and J. W. Moffat, astro-ph/0203164.

[12] C. M. Will, Theory and Experiment in Gravitational Physics, Cambridge University Press, Cambridge, 1991. 
[13] S. Perlmutter et al. Ap. J. 483, 565 (1997), astro-ph/9608192; A. G. Riess, et al. Astron. J. 116, 1009 (1998), astro-ph/9805201; P. M. Garnavich, et al. Ap. J. 509, 74 (1998), astro-ph/9806396; S. Perlmutter et al. Ap. J. 517, 565 (1999), astro-ph/9812133; A. G. Riess, et al. to be published in Ap. J., astro-ph/0104455.

[14] B. Ratra and P. J. E. Peebles, Phys. Rev. D37, 3406(1988); R. R. Caldwell, R. David, and P. J. Steinhardt, Phys. Rev. Lett. 80, 1582 (1998), astro-ph/9708069; I. Zlatev and P. J. Steinhardt, Phys. Lett. B459, 570 (1999), astro-ph/9906481; I. Zlatev, L. Wang, and P. J. Steinhardt, Phys. Rev. Lett. 82, 896 (1999), astro-ph/9807002; L. Wang, R. R. Caldwell, J. P. Ostriker, and P. J. Steinhardt, Ap. J. 530, 17 (2000), astro-ph/9901388. astro-ph/0012539.

[15] S. Carroll, Phys. Rev. 81, 3067 (1998), astro-ph/9806099; astro$\mathrm{ph} / 0107571$.

[16] S. Hellerman, N. Kaloper, and L. Susskind, hep-th/0104180; W. Fischler, A. Kashani-Poor, R. McNees, and S. Paban, hep-th/0104181; E. Witten, "Quantum Gravity in de Sitter Space," Talk at the Strings 2001 Conference, Tata Institute, Mumbai, India, January 2001, http://theory.tifr.res.in/strings/f R. Bousso, JHEP0011, 038 (2000), hep-th/0010252.

[17] C. Csaki, N. Kaloper and J. Terning, hep-ph/0112212 v1; hepph/0112212; C. Deffayet, D. Harari, J. P. Uzan and M. Zaldarriaga, hep-ph/0112118; J. Erlich and C. Grojean, hep-ph/0111335.

[18] K. Freese and M. Lewis, astro-ph/0201229; Chung and Freese, Phys. Rev. D61, 023511 (2000), hep-th/9906542; P. Binetruy, C. Deffayet and D. Langlois, Nucl. Phys. B565, 269 (2000), hep-th/9905012; C. Deffayet, G. Dvali and G. Gabadadze, astro-ph/0105068.

[19] S. Weinberg, Gravitation and Cosmology: Principles and Applications of the General Theory of Relativity, John Wiley \& Sons, 1972 p.418.

[20] J. K. Webb et al., Phys. Rev. Lett. 87, (2001) 091301, astro$\mathrm{ph} / 0012539$. 
[21] C. J. A. P. Martins, A. Melchiorri, R. Trotta, R. Bean, G. Rocha, P. P. Avelino and P. T. P. Viana, astro-ph/0203149; K. M. Nollett and R. E. Lopez, astro-ph/0204325.

[22] T. Banks, M. Dine and M. R. Douglas, Phys. Rev. Lett. 88, (2002) 13130, hep-ph/0112059. 